\title{
The relation between mathematics achievement and spatial reasoning
}

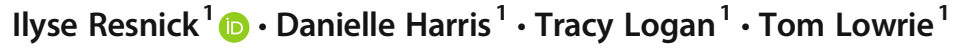

Received: 15 May 2020 / Revised: 15 May 2020 / Accepted: 20 May 2020 /

Published online: 30 May 2020

C) Mathematics Education Research Group of Australasia, Inc. 2020

The articles in this MERJ special issue collectively explore how spatial reasoning is related to, and supports, mathematics learning. Spatial reasoning refers to a suite of different skills that involve the mental manipulation of two-dimensional and threedimensional relations between and within objects. The relation between spatial reasoning and mathematics achievement across development is consistent, predictive, and strengthens over time (Resnick et al. 2019). Fortunately, spatial reasoning can be learned and improved with practice (Lowrie et al. 2018; Uttal et al. 2013), and improvements in spatial reasoning are causally related to improvements in mathematics understanding (Cheng \& Mix 2014; Lowrie et al. 2017). However, these relations vary by task (e.g., Mix et al. 2016), highlighting the need to consider how specific connections are formed and extend to more general performance.

This special issue brings together international research across the fields of mathematics education, development, and cognition, which serves to bridge and bring exposure to frequently siloed findings. In doing so, we (the editorial team) were faced with many challenges reconciling the fundamentally different methodologies, definitions, and valued outcomes each field takes in their research practice. For example, we needed to balance finding appropriate reviewers to ensure the accepted papers would meet the methodological standards within and across the relevant fields of discipline, while purposefully showcasing representative approaches that might complement or be adopted by researchers from other fields. To this end, the articles within the special issue have taken care to explicitly describe their techniques and define their constructs. We have emerged with a collection of nine strong papers across four continents. Contributions range from early career to experienced researchers; such is the breadth and depth of research on this topic.

These papers can be conceptualized along two overarching themes: (1) theoretical/ conceptual links between spatial reasoning and mathematics skills and (2) practical application of spatial reasoning learning in mathematics environments. The Lowrie

Ilyse Resnick

ilyse.resnick@ canberra.edu.au

1 Faculty of Education, University of Canberra, Bruce, ACT 2617, Australia 
et al. 2020 paper is positioned at the nexus of theory and practice, providing a reflection of the field of spatial reasoning within mathematics. Drawing on the research presented within this special issue, the authors suggest future directions build on the respective strengths of mathematics education and psychology. A key aspect of their argument is that spatial reasoning skills do not exist in a vacuum - rather, they are developed and learned in context. Subsequently, spatial assessment, spatial learning programs, and theoretical models would benefit from accounting for context. Mathematics education provides a solid platform to contextualize and foster spatial reasoning.

\section{Contributions to theory}

When characterizing the theoretical connections between spatial reasoning and mathematics achievement, researchers can consider one or both constructs at a broad level (e.g., general mathematics achievement) or at a more detailed level (e.g., a specific mathematics task). Reinhold et al. 2020 examined the role of broad spatial reasoning within a specific mathematics task-complex word problems. To note, like many researchers, Reinhold used a measure of mental rotation (holistically imagining an object rotate) to represent broad spatial reasoning. This is contrasted with Sorby and Panther 2020, who differentiate between mental rotation and what is commonly referred to as spatial visualization (imagining a series of transformations, such as mentally folding sides to make a box). Sorby and Panther 2020 consider the correlations between these two specific spatial reasoning skills with six specific math tasks, as well as composite scores to assess the relation at a broader level.

Another emergent theme within theoretical considerations is the contextualization of spatial reasoning within other cognitive skills (Reinhold et al. 2020), content knowledge (Fujita et al. 2020), and cultural factors (Owens 2020; Sorby \& Panther 2020). Using a generalized linear mixed model, Reinhold et al. 2020 found that students use spatial ability, verbal ability, numerical ability, and general reasoning in solving complex word problems. Despite a male advantage on spatial and numerical ability, the relative unique contributions of the four cognitive skills remain constant across genders. This finding highlights that complex mathematics tasks will likely require coordination of many different cognitive skills (including different spatial reasoning skills) and content knowledge. Indeed, Fujita et al. 2020 observed that domain-specific knowledge was required to coordinate spatial reasoning skills and property-based spatial analytic reasoning. In this study, spatial reasoning alone was not enough to solve geometric problems (that require reasoning about three-dimensional geometrical shapes represented in two dimensions). Future research should aim to disentangle domain knowledge from property-based spatial analytic reasoning and characterize how such coordination takes place.

Owens 2020 considers the contextualized relation between spatial reasoning and mathematics through a cultural, ethnomethodology lens. She draws on many years of research in Papua New Guinea and other studies to describe how Indigenous people embody place and shape in their everyday lives. Owens 2020 shows that the spatial reasoning skills displayed and utilized in most studies provide evidence of understandings related to Western-based mathematics, and 
there may be types of spatial thinking that are not yet widely acknowledged. Despite such cultural differences, Sorby and Panther 2020 find the relation between spatial reasoning supporting mathematics achievement is maintained across cultures. They note that countries with higher mathematics scores similarly have higher scores on the "space and shape" subscale of The Programme for International Student Assessment (PISA).

\section{Practical applications}

When considering the practical implications of how to use spatial learning to support mathematics education, researchers may consider factors at the curriculum level (e.g., how can lessons be designed to support spatial reasoning with mathematics tasks), educator level (e.g., how do educator's spatial reasoning skills influence their teaching), and student level (e.g., how do individual differences in student's spatial reasoning influence their understanding of mathematics tasks). Professional learning as a mechanism for fostering spatial reasoning opportunities in the classroom has emerged within the special issue as a critical aspect of program design. The professional learning has taken the form of explicit pedagogical (Patahuddin et al. 2020) or content (Mulligan et al. 2020; Pollitt et al. 2020) instruction to support spatialized mathematics development. Spatializing established curriculum aides in balancing the demands of an already jampacked curriculum (contrasted with spatial training added as separate lessons) and again emphasizes how spatial reasoning is naturally contextualized within content. Pollitt et al. 2020 found many educators possessed misconceptions or were unaware of spatial reasoning, highlighting the importance of professional learning approaches.

Findings from the Mulligan et al. 2020, Patahuddin e al. 2020, and Pollitt et al. 2020 suggest that it may require a lot of educator support to translate research within the classroom. Educational materials (e.g., lesson plans, assessment activities) and continued support (e.g., researcher meetings) were provided across all three studies. Despite extensive professional learning and detailed lesson plans, Patahuddin et al. 2020 found that classroom-based implementation differed considerably, and Pollitt et al. 2020 found many educators had difficulty implementing the intervention as the semester progressed.

Seah and Horne 2020 and Fujita et al. 2020 both took a student-level approach, characterizing how students used spatial reasoning to complete different geometric mathematics tasks. In both studies, researchers coded student performance on mathematics tasks for correctness as well as spontaneous engagement in spatial reasoning (e.g., their explanation of how they solved the problem included reference to spatial visualization). Correct responses in both studies were supported by spatial reasoning and knowledge of the properties of three-dimensional objects. The authors offered discussion on how this relation could be supported by mathematics education. Their papers suggest the relations between spatial and geometric reasoning continues to be an area of concern in mathematics teaching and learning and that further research needs to consider how to better make those connections.

We hope that the papers within this special issue provide a path forward for applied, integrated studies of spatial reasoning that retain mathematics integrity and experimental soundness with sufficient scale to provide generalizability and impact. 


\section{References}

Cheng, Y.-L., \& Mix, K. S. (2014). Spatial training improves children's mathematics ability. Journal of Cognition and Development, 15(1), 2-11.

Fujita, T., Kondo, Y., Kumakura, H., Kunimune, S., \& Jones, K. (2020). Spatial reasoning skills about 2D representations of 3D geometrical shapes in grades 4 to 9. Mathematics Education Research Journal. https://doi.org/10.1007/s13394-020-00335-w.

Lowrie, T., Logan, T., Harris, D., \& Hegarty, M. (2018). The impact of an intervention program on students' spatial reasoning: student engagement through mathematics-enhanced learning activities. Cognitive Research: Principles and Implications, 3(50), 1-10.

Lowrie, T., Logan, T., \& Ramful, A. (2017). Visuospatial training improves elementary students' mathematics performance. British Journal of Education Psychology, 87, 170-186.

Lowrie, T., Resnick, I., Harris, D., \& Logan, T. (2020). In search of the mechanisms that enable transfer from spatial reasoning to mathematics understanding. Mathematics Education Research Journal. (in press).

Mix, K. S., Levine, S. C., Cheng, Y., Young, C., Hambrick, D. Z., Ping, R., \& Konstantopoulos, S. (2016). Separate but correlated: the latent structure of space and mathematics across development. Journal of Experimental Psychology: General, 145(9), 1206-1227.

Mulligan, J., Woolcott, G., Mitchelmore, M., Busatto, S., Lai, J., \& Davis, B. (2020). Evaluating the impact of a Spatial Reasoning Mathematics Program (SRMP) intervention in the primary school. Mathematics Education Research Journal. https://doi.org/10.1007/s13394-020-00324-z.

Owens, K. (2020). Transforming the established perceptions of visuospatial reasoning: integrating an ecocultural perspective. Mathematics Education Research Journal. (in press).

Patahuddin, S. M., Rokhmah, S. \& Ramful, A. (2020). What does teaching of spatial visualisation skills incur: an exploration through the visualise-predict-check heuristic. Mathematics Education Research Journal. https://doi.org/10.1007/s13394-020-00321-2.

Pollitt, R., Cohrssen, C., Seah, W. T. (2020). Assessing spatial reasoning during play: Educator observations, assessment and curriculum planning. Mathematics Education Research Journal. (in press).

Reinhold, F., Hofer, S., Berkowitz, M., Strohmaier, A., Scheuerer, S., Loch, F., Vogel-Heuser, B., Reiss, K. (2020). The role of spatial, verbal, numerical, and general reasoning abilities in complex word problem solving for young female and male adults. Mathematics Education Research Journal. https:/doi. org/10.1007/s13394-020-00331-0.

Resnick, I., Newcombe, N. S., \& Jordan, N. C. (2019). The relation between spatial reasoning and mathematics achievement in children with mathematics learning difficulties. In P. Rasanen (Ed.), International handbook of mathematical learning difficulties. Springer.

Seah, R. T. K., Horne, M. (2020). The influence of spatial reasoning on analysing about measurement situations. Mathematics Education Research Journal. https://doi.org/10.1007/s13394-020-00327-w.

Sorby, S. A., Panther, G. C. (2020). Is the key to better PISA math scores improving spatial skills?. Mathematics Education Research Journal. https://oi.org/10.1007/s13394-020-00328-9.

Uttal, D. H., Meadow, N. G., Tipton, E., Hand, L. L., Alden, A. R., Warren, C., \& Newcombe, N. S. (2013). The malleability of spatial skills: a meta-analysis of training studies. Psychological Bulletin, 139(2), 352402 .

Publisher's note Springer Nature remains neutral with regard to jurisdictional claims in published maps and institutional affiliations. 